\title{
Free-Volume and Tensile Properties of Glass Fibre Reinforced Polyamide 6 Composites
}

\author{
E. DRYZEK ${ }^{a, *}$, M. WRÓBEL ${ }^{b}$ AND E. JUSZYŃSKA-GAŁĄZKA ${ }^{a}$ \\ ${ }^{a}$ Institute of Nuclear Physics, Polish Academy of Sciences, PL-31342 Kraków, Poland \\ ${ }^{b}$ AGH University of Science and Technology, al. Mickiewicza 30, 30-059 Kraków, Poland
}

\begin{abstract}
The tensile properties and free volume of commercially available modified polyamid 6 and polyamid 6 composites with 15 and $30 \mathrm{wt} \%$ of glass fibre were the subject of the studies. The tensile test allowed us to obtain the stress-strain curves and determine the tensile properties of the polyamid 6 samples. The positron lifetime measurements were performed for the samples before the test and for the samples in the vicinity of the break after they failed. The composites exhibited slightly lower values of the ortho-positronium lifetime and therefore smaller size of the local free volumes in comparison to polyamid 6 without reinforcement. The analysis of the positron lifetime spectra indicated size distribution of the free volume. The initially narrower distributions for the composite samples became broader as a result of the deformation. The deformation caused also increase of the ortho-positronium intensity in the obtained positron lifetime spectra.
\end{abstract}

DOI: 10.12693/APhysPolA.132.1501

PACS/topics: 78.70.Bj, 61.41.+e

\section{Introduction}

Polyamide 6 (PA6) is one of the basic engineering polymer materials. It is a thermoplastic polymer with good chemical resistance to nonpolar solvents, excellent thermal properties and very good mechanical properties. Its mechanical properties can be easily modified by using suitable fillers. For example PA6 reinforced with glass fibre (GF) in amount higher than $40 \mathrm{wt} \%$ is a good metal replacement.

It has been well established that macroscopic properties such as tensile strength and Young's modulus depend on microstructural properties of polymers and polymer composites. In case of the latter which are strongly heterogeneous systems the external load causes micromechanical deformation processes determined to a large extent by the properties of the matrix polymer. One of the factors taken into account while considering the mechanisms of inelastic deformation in amorphous polymers is the presence of free volume. The volume excess is an important factor that enables local shear transformations $[1,2]$. The local free volume properties of the material can be determined directly by positron annihilation lifetime spectroscopy (PALS). A positron injected into polymer can form a bound state with an electron called positronium $(\mathrm{Ps})$. Ps is created in low electron density regions like local free volume holes. There can be formed two spin states of Ps: para-Ps ( $p$-Ps) and ortho-Ps (o-Ps) due to possible positron and electron spin alignment, i.e., antiparallel and parallel, respectively. In vacuum, the self-annihilation lifetime of $o$-Ps is $142 \mathrm{~ns}$ and it decays into three quanta while the $p$-Ps lifetime is much shorter,

*corresponding author; e-mail: ewa.dryzek@ifj.edu.pl i.e., 125 ps with decay into two quanta. In molecular solids, due to its relatively long lifetime, o-Ps interacts with the electrons of surrounding molecules. Its lifetime is determined mainly by the annihilation of the positron with one of these external electrons. This process is called pick-off annihilation. It causes shortening of the o-Ps lifetime to several ns. The positron annihilation lifetime spectrum measured for polymers usually contains at least three exponential decay components, which can be ascribed to annihilation of $p$-Ps, free positrons and $o$-Ps annihilating in the pick-off process, respectively. Third component $\tau_{3}$ is related to the mean size of local free volume holes $[3,4]$. This relation is used to study changes of the free volume hole size and its distribution caused by the presence of GF reinforcement in PA6 matrix and then deformation of the samples in tensile test.

The attempt to correlate the free volume and tensile properties of GF reinforced PA6 is the aim of the paper. Additionally, differential scanning calorimetry (DSC) and scanning electron microscopy (SEM) were used to characterize the samples.

\section{Experimental details}

The materials under investigation were commercially available PA6 and PA6 composites with 15 and $30 \mathrm{wt} \%$ of glass fibre, i.e. ArtAMID6 15GF and ArtAMID6 430GF. The dogbone/paddle A1 type specimens with $4 \times 10 \mathrm{~mm}^{2}$ cross-section and reduced length of $80 \mathrm{~mm}$ used for tensile test were injection moulded. Samples met the PNEN ISO 527:1998 standard requirements. The tensile tests were performed at room temperature using Tensile Machine Instron 4502 and the strain rate was equal to $5 \mathrm{~mm} / \mathrm{min}$. Before the test samples have been conditioned in natural conditions for about six months at room temperature. The humidity absorption of the material according to the technical data of the producer (ISO 62) 
is $1.6 \%, 1.1 \%$ and $1.0 \%$ for PA6, ArtAMID6 $15 \mathrm{GF}$ and ArtAMID6 430GF, respectively. The change of the mass after drying for $4 \mathrm{~h}$ at $100^{\circ} \mathrm{C}$ indicated similar values of the humidity absorption.

DSC experiments have been performed with the use of a TA DSC 2500 instrument, to obtain glass transition temperatures $T_{g}$, crystallinity, and melting temperatures. The heat flow during cooling and heating of the samples, was equal to 10 and $20^{\circ} \mathrm{C} / \mathrm{min}$ maintained for 5 min at $-90^{\circ} \mathrm{C}$ and $300{ }^{\circ} \mathrm{C}$ (at the end of measured cycles). In the first step, a fresh sample was cooled down to low temperatures and after that a heating up to $300^{\circ} \mathrm{C}$ (a first cycle) took place. Then again a cooling down was applied and the second cycle with a higher rate of temperature change was carried out.

FEI Inspect S50 scanning electron microscope was used for the fracture surface characteristic. For the investigation specimens gold coated on the sputter coater EMITECH K575X were used.

For PALS measurements a lifetime spectrometer with the time resolution (FWHM) of 280 ps was used. A positron source of ${ }^{22} \mathrm{Na}$ deposited on Kapton foil $(7.5 \mu \mathrm{m}$ thick) was sandwiched between two identical samples. The positron lifetime measurements were performed for the as received samples and for the samples in the vicinity of the break after they failed. The obtained PALS spectra with a total number of $2 \times 10^{6}$ counts were analyzed using the LT code in the distribution mode, which assumes that for a determined number of the annihilation channels, the annihilation rate, $\lambda_{i}=1 / \tau_{i}$, follows a log-normal function [5]. The fit of these functions to the spectra provides the positron lifetimes $\tau_{i}$ and their intensities $I_{i}$ as well as the width of the corresponding distribution (standard deviation $\sigma_{i}$ of the lifetime $\tau_{i}$ ). Taking into account three annihilation channels, i.e, $p$ Ps, free positrons and $o$-Ps pick-off annihilation as well as background subtraction and the source contribution allowed us to obtain $\chi^{2}$ of the fit close to unity.

\section{Results and discussion}

\subsection{DSC measurements}

During heating of all samples the endothermic anomalies have been detected on DSC thermograms, for temperature change rates $10^{\circ} \mathrm{C} / \mathrm{min}$ and $20^{\circ} \mathrm{C} / \mathrm{min}$. This allowed us to obtain the melting enthalpy. The obtained values of melting enthalpy as well as the melting temperatures for two measurement cycles are given in Table I. The crystallinity degree was calculated assuming that the enthalpy of $100 \%$ crystalline PA is equal to $190 \mathrm{~J} / \mathrm{g}[6]$. It can be seen from Table I that the PA sample exhibits the lowest initial degree of crystallization. After melting of the samples in first cycle, the degree of crystallinity decreases. The glass transition anomalies, i.e., vitrification on cooling and softening of glassy state on heating, have been also detected. The midpoint temperatures on heating in the first cycle are following: $44.4^{\circ} \mathrm{C}, 47.5^{\circ} \mathrm{C}$, and $44.5^{\circ} \mathrm{C}$ for PA6, PA6 $15 \mathrm{wt} \% \mathrm{GF}$ and PA6 $30 \mathrm{wt} \% \mathrm{GF}$, respectively. This indicates that the samples at room temperature are below $T_{g}$.

TABLE I

The melting temperatures $T_{m}$ and enthalpies $\Delta H$ for PA6 and PA $6+n$ wt $\%$ GF composite samples for the first cycle (fresh samples) and second cycle. The degree of crystallinity was established using the formula $X_{t}=\Delta H_{m} / 190 \mathrm{~J} / \mathrm{g}$ [7].

\begin{tabular}{c|c|c|c|c|c|c}
\hline \hline \multirow{2}{*}{$n$} & \multicolumn{2}{|c|}{ First cycle $\left(10 \frac{{ }^{\circ} \mathrm{C}}{\mathrm{min}}\right)$} & \multicolumn{3}{|c}{ Second cycle $\left(20 \frac{{ }^{\circ} \mathrm{C}}{\mathrm{min}}\right)$} \\
\cline { 2 - 7 } & $T_{m}$ & $\Delta H$ & Cryst. & $T_{m}$ & $\Delta H$ & Cryst. \\
& {$\left[{ }^{\circ} \mathrm{C}\right]$} & {$[\mathrm{J} / \mathrm{g}]$} & {$[\mathrm{wt} \%]$} & {$\left[{ }^{\circ} \mathrm{C}\right]$} & {$[\mathrm{J} / \mathrm{g}]$} & {$[\mathrm{wt} \%]$} \\
\hline PA & 220 & 38.2 & 20 & 213 & 36 & 19 \\
15 & 215 & 82 & 43 & 219.4 & 50.8 & 27 \\
30 & 212 & 77.8 & 41 & 218.3 & 28.9 & 15
\end{tabular}

\subsection{Tensile test}

The tensile test showed a significant increase in strength and modulus and decrease in plasticity due to the GF addition, see Fig. 1 and Table II. This is due to a relatively high modulus and strength of the fibreglass, i.e. in the range 65-90 $\mathrm{GPa}$ and 3.4-4.9 $\mathrm{GPa}$, respectively, then the effect observed in the present study is typical for the GF reinforced composites [7].

TABLE II

Tensile properties of PA6 and PA6 $+n$ wt $\%$ GF composite samples.

\begin{tabular}{c|c|c|c|c}
\hline \hline \multirow{2}{*}{$\begin{array}{c}n \\
{[\%]}\end{array}$} & Modulus & $\begin{array}{c}\text { Yield } \\
\text { stress }\end{array}$ & $\begin{array}{c}\text { Tensile } \\
\text { strength }\end{array}$ & $\begin{array}{c}\text { Strain } \\
\text { at break } \\
{[\mathrm{mm} / \mathrm{mm}]}\end{array}$ \\
\cline { 2 - 4 }$\left[\begin{array}{c}\mid 3 \\
\text { PA }\end{array}\right.$ & 760 & 43 & 48 & 2.42 \\
15 & 1910 & 74 & 74 & 0.15 \\
30 & 3320 & 84 & 83 & 0.06
\end{tabular}

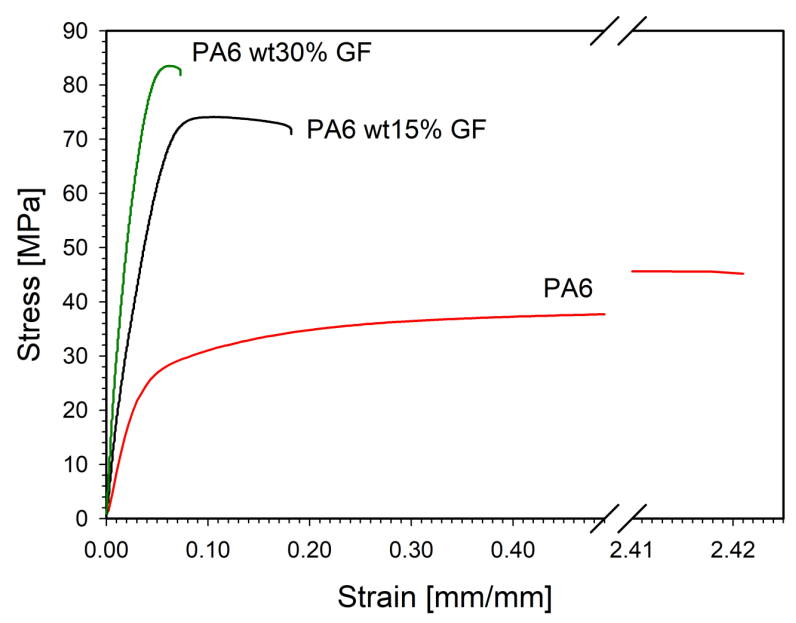

Fig. 1. Stress-strain curves for the PA6 and PA6 composite samples obtained at room temperature with strain rate $5 \mathrm{~mm} / \mathrm{min}$. 


\subsection{SEM observations of fracture surface}

SEM micrographs of fracture surface in Fig. 2 show ductile areas, i.e., (a) for PA6 $15 \mathrm{wt} \% \mathrm{GF}$ and (b) for PA6 30wt\% GF, and brittle areas, i.e., (c) for PA6 $15 \mathrm{wt} \% \mathrm{GF}$ and (d) for PA6 30wt\% GF. The first exhibits high plastic deformation of the matrix. High ductility may be caused by water absorption $[8,9]$. The lack of matrix residues on the surface of the pulled out fibres indicates failure of the interface between matrix and fibres. For the brittle fraction the matrix deformation is smaller. The pulled out fibres are also visible.

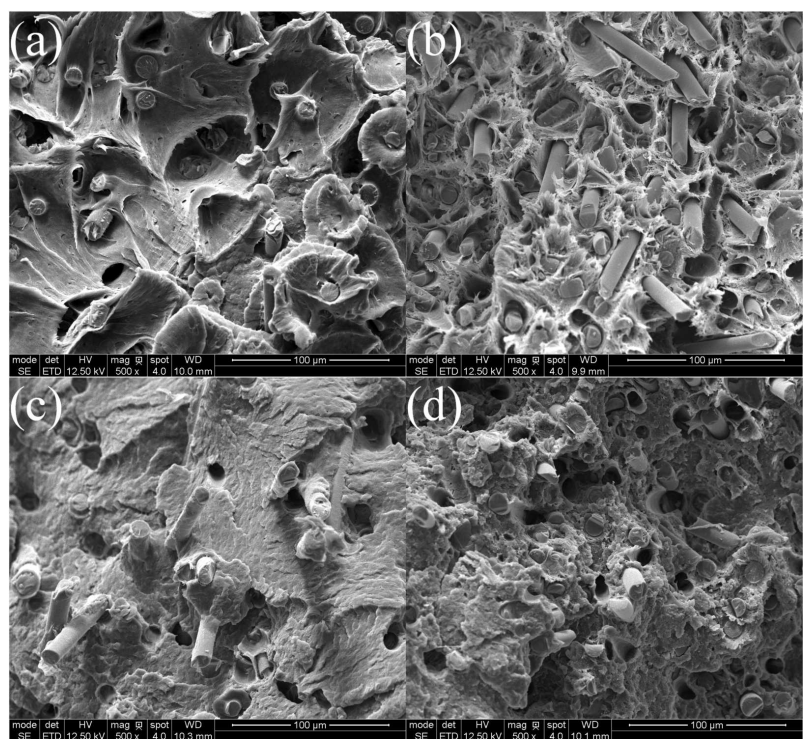

Fig. 2. SEM micrographs of tensile fracture surface for PA6 15 wt\% GF in parts (a) and (c), and for PA6 $30 \mathrm{wt} \%$ GF in parts (b) and (d).

\subsection{PALS measurements}

With the assumption that pick-off is the main process of $o$-Ps annihilation, i.e. other quenching mechanisms of $o$-Ps such as ortho-para spin conversion in internal or external magnetic fields or chemical quenching are negligible, i.e. $\tau_{3}=\tau_{\text {pick-off }}$, average radius of free volume holes can be calculated according to the semi-empirical model $[3,4]$. The model relates the pick-off lifetime $\tau_{3}$ to the average free volume radius of a spherical hole by the following equation:

$$
\tau_{3}=\frac{1}{2}\left(1-\frac{R}{R+\Delta R}+\frac{1}{2 \pi} \sin \frac{2 \pi R}{R+\Delta R}\right)^{-1},
$$

where $R$ is a radius of the hole and $\Delta R$ is an electron layer thickness. The value of $\Delta R$ was estimated as $1.66 \AA$.

In the performed analysis of the spectra, distributions of the annihilation rate, $\xi\left(\frac{1}{\tau_{3}}\right)$, were obtained. Then distributions of the radii $f(R)$ of the free volume holes, i.e. probability density function, can be calculated using the following equation:

$$
f(R)=\frac{2 \Delta R}{R+\Delta R} \cos \left(\frac{2 \pi R}{R+\Delta R}-1\right) \xi\left(\frac{1}{\tau_{3}}\right) .
$$

It is worth noticing that PALS was applied to study a few issues concerning PA6, i.e., effect of crystallization, water uptake or broader understood microstructure and the PALS spectra were also analyzed in terms of continuous distributions of lifetimes $[6,10,11]$.

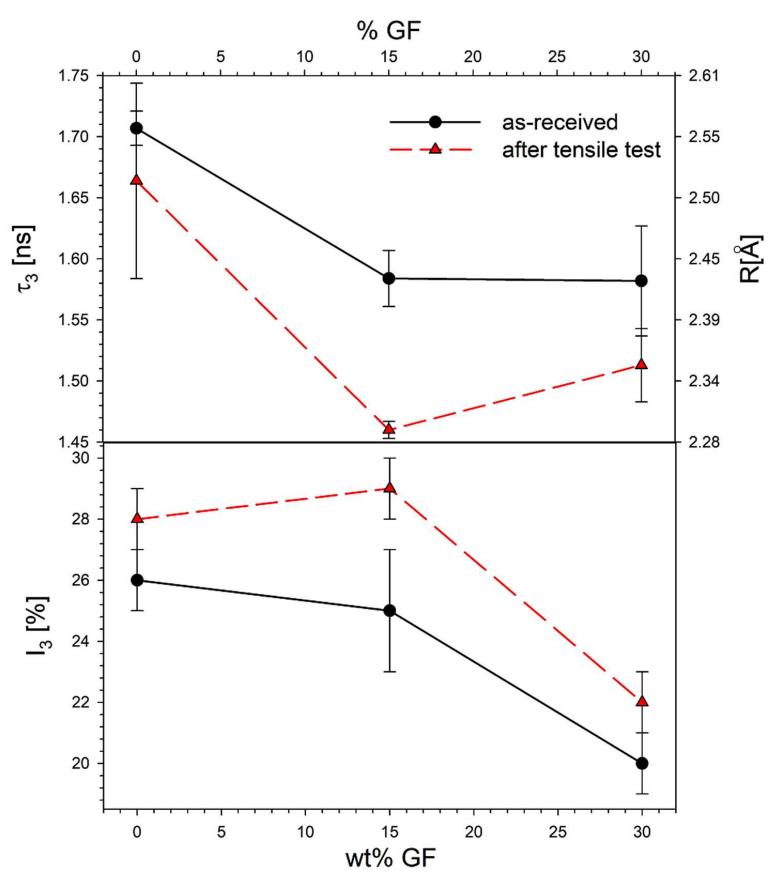

Fig. 3. Positron lifetimes $\tau_{3}$ and its intensities $I_{3}$ for the PA6 and PA6 composite samples. The right axis of the upper figure shows the values of the local free volume hole radii calculated from Eq. (1).

Figure 3 presents values of the mean $o$-Ps lifetime, $\tau_{3}$ (calculated as the mass center of the distribution), and its intensity, $I_{3}$, for PA6 and composite samples as a function of GF amount. It can be seen that addition of the GF reinforcement causes slight decrease of $\tau_{3}$ which indicates decrease of the mean free volume hole size. It is accompanied by the decrease of the intensity $I_{3}$. Changes of the mean $o$-Ps lifetime after the tensile test are most visible for the sample with $15 \mathrm{wt} \% \mathrm{GF}$, for which $\tau_{3}$ decreases and $I_{3}$ increases. For the remaining samples the changes are within the error range but the tendency is the same.

Figure 4 shows the free volume hole radius distributions, $f(R)$. For the as-received samples it is visible that the width of the distribution for the composite samples is much smaller than for the polymer sample. Then the reinforcement particles influence the local microstructure of the polymer. Similar narrowing of the distributions was observed by Awad et al. [12] for zinc oxide/waterborne polyurethane nanocomposites and was ascribed to interfacial interactions between the surface of nanoparticles and polymer matrix. Narrower free volume distribution for short GF reinforced polyethersulfone (PES) was also 


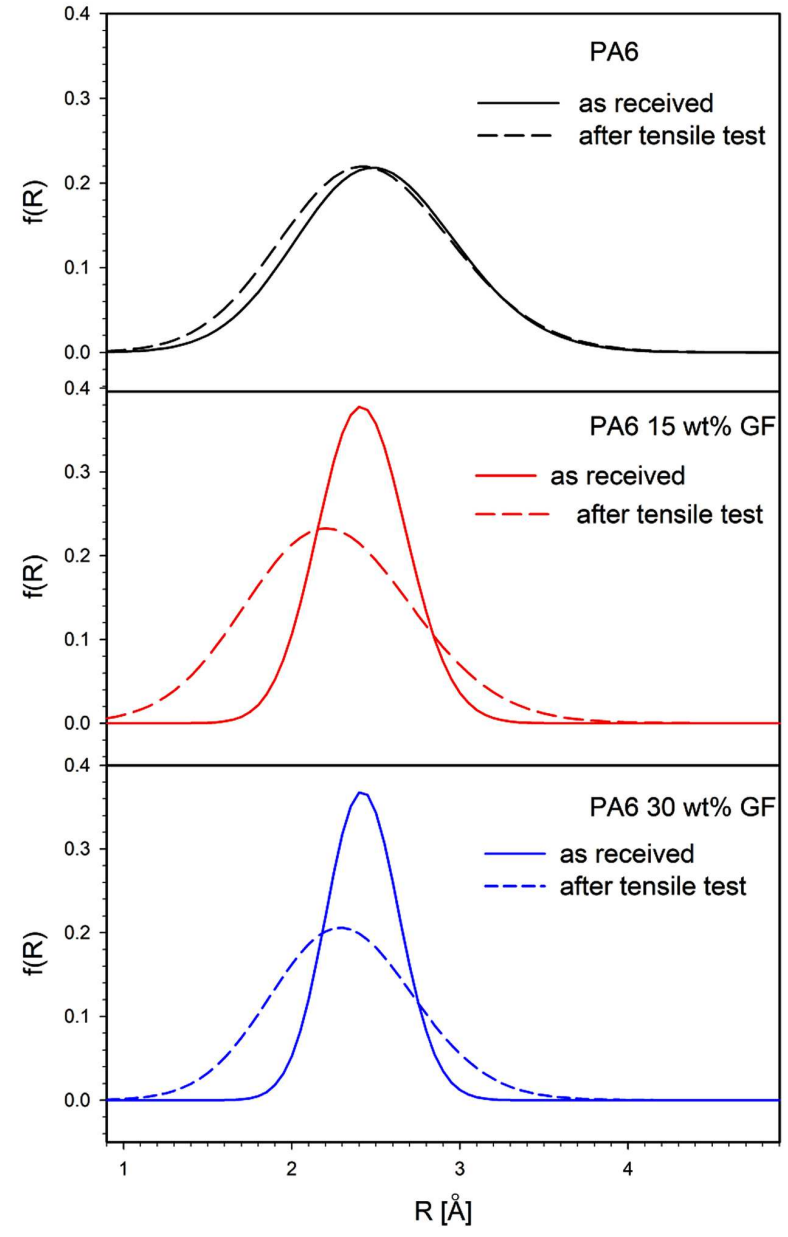

Fig. 4. Distributions of free volume radii for the asreceived PA6 and PA6 composite samples (solid lines) and the samples after the tensile test (dashed lines). PALS measurements were performed in the vicinity of the break after the samples failed.

reported. The decreased positron lifetime parameters were correlated with improved mechanical properties and reduced crystallinity of the composites studied. All this was attributed to the increased chemical and physical interaction between the functional groups of short GF and PES matrix [13]. However, in our case the PA sample has significantly lower degree of crystallinity which may also contribute to broader distribution of the free volume hole radii.

When being uniaxially drawn isotropic semicrystalline polymer systems transform into a highly oriented fibrillar state. Polymer chains become oriented preferentially along the drawing direction. Tensile deformation of PA6 at elevated temperature above $T_{g}$ and for water saturated samples was studied by Men et al. [14]. PA6 as a semicrystalline polymer system can be treated as composed of two interpenetrating networks: the hard crystalline phase and the soft amorphous phase. The low deformation properties are determined by the crystalline phase due to the activation of the intralamellar block slip mechanism. For large deformations the deformation mechanism changes from the block slip mechanism to a stress-induced melting-recrystallization process. Tensile deformation of bulk PA6 in the preyield strain was studied by Millot et al. by combined in situ wide-angle and small-angle X-ray scattering [15]. Structural changes involved by plastic deformation may influence also the local free volume features.

As it was mentioned above changes of the PALS parameters, i.e., $\tau_{3}$ and $I_{3}$ for the PA6 sample after the tensile test are small. The width of the free volume radius distribution also does not change significantly as it can be seen in Fig. 4. Therefore, it seems that the free volume microstructure generated by the large deformation differs from the initial microstructure only to a small degree. However, distributions obtained for the GF reinforced PA6 composite samples after the tensile test are significantly broader than those for the as-received samples and their width is similar to that for the PA6 polymer without reinforcement. Such a behavior indicates that after the large deformation the influence of GF reinforcement on the free volume microstructure of the polymer matrix weakens which may be a consequence of damage within the interface and loss of adhesion between the polymer matrix and GF reinforcement. As a result the interface does not fulfill its role.

\section{Conclusions}

In the present work we have investigated the influence of the GF reinforcement on the free volume microstructure of PA6 and its changes caused by tensile deformation. We found that the composite samples exhibited lower values of the mean $o$-Ps lifetime indicating decrease of the mean local free volume size. It is accompanied by narrowing of the local free volume distribution. The initially narrower distributions for the composite samples become broader as a result of deformation and their width is comparable to that for PA6 sample. The deformation causes also increase of the $o$-Ps intensity in the obtained positron lifetime spectra. These changes indicate that alterations in the PA6 matrix free volume microstructure are connected to damage within the interface and loss of adhesion between the polymer matrix and GF reinforcement.

\section{Acknowledgments}

We would like to thank Dr. A. Ziółkowski from Art Plast Company, Poniatowa, Poland who provided composite samples used in the present study.

\section{References}

[1] A.S. Argon, V.V. Butalov, P.H. Mott, U.W. Suter, J. Rheol. 39, 377 (1995).

[2] S. Goyanes, G. Rubiolo, W. Salgueiro, A. Somoza, Polymer 46, 9081 (2005). 
[3] S.T. Tao, J. Chem. Phys. 56, 5499 (1972).

[4] N. Eldrup, D. Lightbody, J.N. Sherwood, Chem. Phys. 63, 51 (1981).

[5] J. Kansy, Nucl. Instrum. Methods Phys. Res. A 374, 235 (1996).

[6] M. Dębowska, J. Pigłowski, C. Ślusarczyk, P. Schmidt, J. Rudzińska-Girulska, T. Suzuki, R. Yu, W. Biniaś, Fibres Text. East. Eur. 13, 64 (2005).

[7] Data Book-JPS Composite Materials, JPS Industries Inc. Company, US.

[8] N. Sato, T. Kurauchi, S. Sato, O. Kamigaito, J. Mater. Sci. 26, 3891 (1991).

[9] S. Mortazavian, A. Fatemi, A. Khosrovaneh, $S A E$ Int. J. Mater. Manuf. 8, 435 (2015).
[10] G. Dlubek, F. Redmann, R. Krause-Rehberg, J. Appl. Polym. Sci. 84, 244 (2002).

[11] G. Dlubek, M. Stolp, Ch. Nagel, H.M. Fretwell, M.A. Alam, H.-J. Radusch, J. Phys. Condens. Matter 10, 10443 (1998).

[12] S. Awad, H. Chen, X. Gu, J.L. Lee, E.E. Abdel-Hady, Y.C. Jean, Macromolecules 44, 29 (2011).

[13] L.M. Munirathnamma, H.B. Ravikumar, J. Appl. Polym. Sci. 133, (2016).

[14] Y. Men, J. Rieger, K. Hong, J. Polym. Sci. B Polym. Phys. 43, 87 (2005).

[15] C. Millot, R. Séguéla, O. Lame, L.-A. Fillot, C. Rochas, P. Sotta, Macromolecules 50, 1541 (2017). 